\title{
Cell-type, allelic, and genetic signatures in the human pancreatic beta cell transcriptome
}

\author{
Alexandra C. Nica, ${ }^{1,2,3}$ Halit Ongen, ${ }^{1,2,3}$ Jean-Claude Irminger, ${ }^{1,2}$ Domenico Bosco, ${ }^{4}$ \\ Thierry Berney, ${ }^{4}$ Stylianos E. Antonarakis, ${ }^{1,2}$ Philippe A. Halban, ${ }^{1,2,5}$ \\ and Emmanouil T. Dermitzakis ${ }^{1,2,3,5}$
}

${ }^{1}$ Department of Genetic Medicine and Development, University of Geneva Medical School, Geneva 1211, Switzerland; ${ }^{2}$ Institute of Genetics and Genomics of Geneva (iGE3), Geneva 1211, Switzerland; ${ }^{3}$ Swiss Institute of Bioinformatics, Geneva 1211, Switzerland; ${ }^{4}$ Cell Isolation and Transplant Center, Department of Surgery, Geneva University Hospitals and University of Geneva, Geneva 1211 , Switzerland

\begin{abstract}
Elucidating the pathophysiology and molecular attributes of common disorders as well as developing targeted and effective treatments hinges on the study of the relevant cell type and tissues. Pancreatic beta cells within the islets of Langerhans are centrally involved in the pathogenesis of both type 1 and type 2 diabetes. Describing the differentiated state of the human beta cell has been hampered so far by technical (low resolution microarrays) and biological limitations (whole islet preparations rather than isolated beta cells). We circumvent these by deep RNA sequencing of purified beta cells from 11 individuals, presenting here the first characterization of the human beta cell transcriptome. We perform the first comparison of gene expression profiles between beta cells, whole islets, and beta cell depleted islet preparations, revealing thus beta-cell-specific expression and splicing signatures. Further, we demonstrate that genes with consistent increased expression in beta cells have neuronal-like properties, a signal previously hypothesized. Finally, we find evidence for extensive allelic imbalance in expression and uncover genetic regulatory variants (eQTLs) active in beta cells. This first molecular blueprint of the human beta cell offers biological insight into its differentiated function, including expression of key genes associated with both major types of diabetes.
\end{abstract}

[Supplemental material is available for this article.]

Phenotypic differences among cell types, individuals, and populations (Stranger et al. 2007; Dimas et al. 2009; Nica et al. 2011) are determined by variation in gene expression. A substantial proportion of this variability is driven by DNA polymorphisms residing in regulatory elements proximal or distal to the affected genes (Price et al. 2011; Grundberg et al. 2012). Numerous such variants have now been mapped for a variety of tissues, highlighting their tissue dependent properties and hence the acute need for expression profiling of a diverse panel of cell types (Nica et al. 2011; Grundberg et al. 2012). This became even more evident in the context of functionally elusive results from genome-wide association studies (GWAS), as transcript abundance has been shown to provide a direct and causal link between genotype and disease susceptibility (Emilsson et al. 2008; Nica et al. 2010). This connection has been mostly attainable in disease-relevant tissues, often in concordance with our present knowledge about the etiology of complex diseases (Nica et al. 2011; Grundberg et al. 2012). With the substantial improvement in the accuracy and resolution of transcriptome profiling by direct RNA sequencing (RNA-seq) (Montgomery et al. 2010; Pickrell et al. 2010), it is now possible to explore these relations comprehensively in an unbiased manner, with no theoretical limitation for dynamic range of expression detection provided there is sufficient sequencing depth.

\footnotetext{
${ }^{5}$ Corresponding authors

E-mail emmanouil.dermitzakis@unige.ch

E-mail Philippe.Halban@unige.ch

Article published online before print. Article, supplemental material, and publication date are at http://www.genome.org/cgi/doi/10.1101/gr.150706.112.
}

Insulin-secreting pancreatic beta cells within the islets of Langerhans have been consistently involved in the pathogenesis of diabetes via autoimmune mediated apoptosis (type 1 diabetes; T1D) (Tisch and McDevitt 1996) or insulin deficiency (type 2 diabetes; T2D) (Saltiel and Kahn 2001). The genetic landscape of both common forms of the disease has been substantially broadened, with now over 60 known loci robustly associated with either type 1 (Barrett et al. 2009) or type 2 diabetes (Morris et al. 2012). As already attested (Gaulton et al. 2010), regulatory changes will likely explain a proportion of these associations, but uncovering them is entirely dependent on first describing the transcriptional profile of the beta cell and understanding its genetic determinants. In this context, we interrogate here the human beta cell transcriptome in multiple whole-genome sequenced individuals and uncover beta-cell-specific features in the context of other pancreatic endocrine cell types.

\section{Results}

Following ethical guidelines at the University Hospital in Geneva, we obtained human islets from 11 cadaveric pancreata from individuals without documented diabetes (description in Supplemental Table 1). The islet preparations were of high purity (mean \pm SD: $84.6 \pm 10.3 \%$ ) as measured by dithizone staining, indicating little contamination with exocrine tissue. The islet cells were sorted by fluorescence-activated cell sorting (FACS) as previously documented (Parnaud et al. 2008) in order to obtain a highly purified population of fully functional beta cells for each individual: $86.7 \pm 6.8 \%$ beta cell purity assessed by immunofluorescence staining for insulin (INS). We identified any contaminating alpha 
and delta cells by costaining for glucagon (GCG) $(4.4 \pm 3.4 \%)$ and somatostatin $(S S T)(2.7 \pm 0.8 \%)$. For five of the samples, we also generated beta cell depleted "nonbeta" populations consisting primarily of glucagon secreting alpha cells $(59.8 \pm 14.1 \%)$, with $4.3 \pm 2.6 \%$ beta cells. RNA was extracted immediately after FACS sorting from 23 cell preparations (11 beta, seven islet, and five nonbeta), cDNA libraries constructed (Thomas and Ansel 2010), and sequenced at very high depth.

We obtained a median of 209 million total reads (paired end, 49 bases) per sample (Supplemental Fig. 1a), attaining thus an exceptional transcriptome resolution. The reads were mapped to the latest version of the human genome assembly (GRCh37/hg19) using BWA (Li and Durbin 2009) and subsequently filtered for mapping quality and correct pairing. On average, 75\% of the filtered reads (median 121 million reads per sample) mapped to known exons annotated by GENCODE (version 10) (Harrow et al. 2006). We quantified all genes expressed in $>90 \%$ of individuals in either of the three cell type preparations $(N=19,975)$, normalizing the read counts to exonic gene length and sequencing depth (reads per kilobase per million [RPKM] mapped reads) (Mortazavi et al. 2008).

Principal component analysis (PCA) on RPKM units (Fig. 1A) indicates that beta cells and islets cluster closely together and markedly separate from nonbeta cells, with INS, insulin-like growth factor 2 (IGF2), GCG, transthyretin (TTR), regenerating islet-derived 1 alpha (REG1A), and SST driving most of this separation (Supplemental Fig. 2). We notice a clear clustering of the islet-derived RNA-seq data in the context of 18 other tissues (obtained from Ambion's FirstChoice Human Total RNA Survey Panel and processed alike), with liver bearing most similarity to the islet samples. Unsurprisingly, islet purity influences gene expression (lowest purity preparation P786i clusters less well), yet this effect is not very large in our samples of overall high quality. To illustrate this, we quantified the proportion of true positives estimated from the enrichment of significant $P$-values (pi1) (Storey and Tibshirani 2003) resulting after correlating each gene's RPKM with purity (pi1 =0-0.2) (Supplemental Fig. 3).

Overall, we observe a high ranked correlation between beta cell and islet-expressed genes (rho $=0.94)($ Fig. 1B), and we estimate that $87 \%$ of the variance in beta cell gene expression can be explained by using islet expression as proxy. Given the estimated
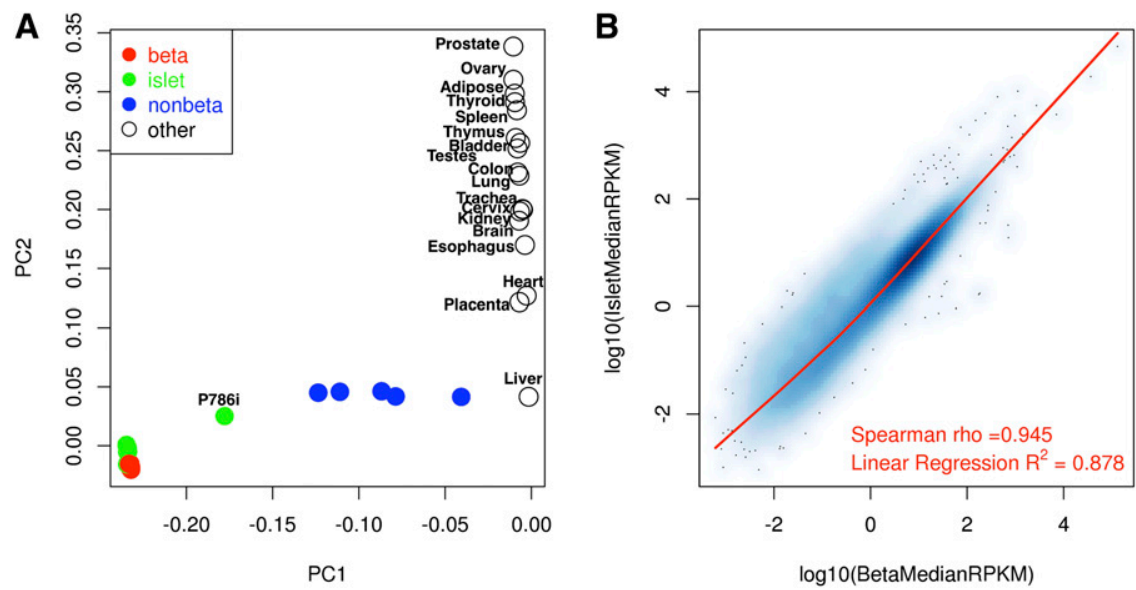

Figure 1. High overall similarity between beta cell and islet gene expression. ( $A$ ) PCA analysis of gene RPKMs for beta $(N=11)$, islet $(N=7)$, nonbeta $(N=5)$ preparations, and 18 other tissues from unrelated individuals. Beta cell and islet samples cluster together, separating from nonbetas. The other tissues cluster separately, with liver being the most similar to the islet-derived RNA-seq data. (B) Scatterplot of beta cell versus islet median RPKMs on $\log _{10}$ scale. $\sim 75 \%$ beta cell composition of the human islet (Pisania et al. 2010) and the quality of the material used for this study, the similarity between these two cell types is not surprising. As expected, INS was by far the most abundantly transcribed gene, followed by INS-IGF2 and $I G F 2$, making up $\sim 38 \%, 10 \%$, and $2 \%$ of the total nuclear beta cell transcriptome, respectively (Fig. 2A). The corresponding relative percentages are lower in the islet, by approximately twofold (INS 21\%, INS-IGF2 5.8\%, IGF2 1.1\%).

To uncover transcriptional signatures specific to beta cells, we next tested for differential gene expression after appropriate statistical modeling of the raw counts (DESeq) (Anders and Huber 2010). When comparing the beta cell sequence count data to 18 non-islet tissues, we discovered 2980 differentially expressed (DE) genes ( $\sim 12 \%$ of genes tested) at $10 \%$ false discovery rate (FDR), 417 of which were significantly overexpressed in beta cells. These genes underlie the general features of endocrine pancreatic activity (DAVID) (Huang et al. 2009; Supplemental Table 2, enrichment results). We next sought to identify beta-cell-specific genes only in the context of the islet and its other hormone-producing cell types, while controlling for islet purity (included as covariate). Of the 19,975 genes tested, we found $5555 \mathrm{DE}$ genes between the beta cell and islet preparations and $4380 \mathrm{DE}$ genes when comparing beta and nonbeta cells (Table 1A). The difference in power is due to the smaller sample size of the nonbeta population and its greater variance in composition of cell types. We find in both cases a larger proportion (approximately two-thirds) of overexpressed genes in the more heterogeneous of the cell populations compared $\left(\log _{2}\right.$ Fold Change $\left.>0\right)$ (Fig. 2B,C). The remaining one-third of the DE genes are significantly overexpressed in beta cells, with smaller fold changes in islets as expected ( $\log _{2}$ Fold Change mean $\pm \mathrm{SD}$ : $-1.06 \pm 0.37$ islet/beta, $-1.64 \pm 0.87$ nonbeta/beta). Notably, we observe a significant enrichment of annotated lincRNAs in beta cell overexpressed genes, corroborating their cell-type-specific expression properties reported previously in other tissues (Cabili et al. 2011): We detect 132 overexpressed lincRNAs in beta versus islet (1.59-fold enrichment over protein coding genes, Fisher's $P$-value: $\left.1.1 \times 10^{-4}\right)$ and 148 overexpressed lincRNAs in beta versus nonbeta (1.9-fold enrichment, Fisher's $P$-value: $1.02 \times 10^{-7}$ ). These are likely underestimates as we limited ourselves to noncoding RNAs currently present in the annotation (Morán et al. 2012).

To portray the unique biological characteristics of beta cells in the islet context, we defined a stringent "beta cell specific" set of genes $(N=526)$ as the intersection of the beta cell overexpressed genes from the two pairwise comparisons (1987 beta-islet and 1583 beta-nonbeta) ranking in the following order of expression: beta > islet > nonbeta. Similarly, we defined a set of "nonbeta cell specific" genes $(N=614)$ consistently and significantly underexpressed in beta cells (nonbeta $>$ islet $>$ beta). A functional annotation analysis reveals that beta-cellspecific genes have neuron-like properties (Table 2 ), a similarity noted earlier in studies implicating glucose-sensing hypothalamic neurons in nutrient homeostasis (Schwartz et al. 2000) or pancreatic hormone release (Ahren 2000). Nonbetacell-specific genes are mostly enriched for cell surface components (N-glycopro- 
A

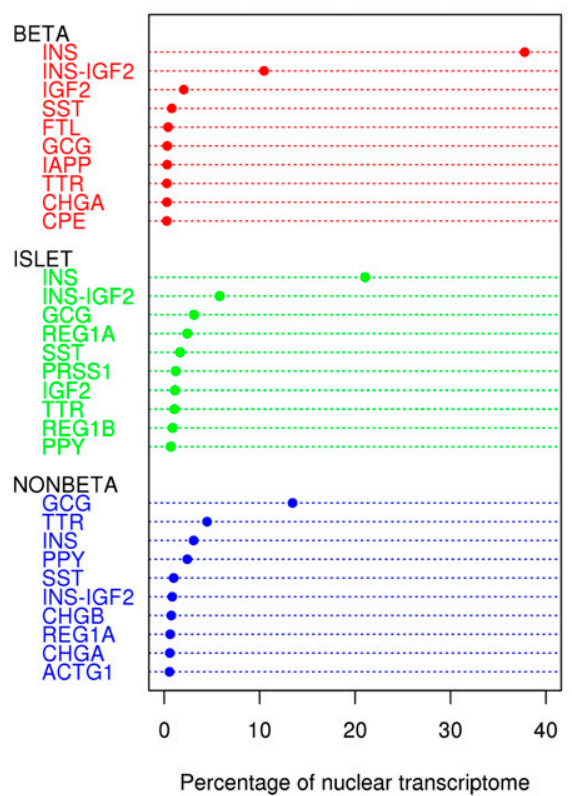

B

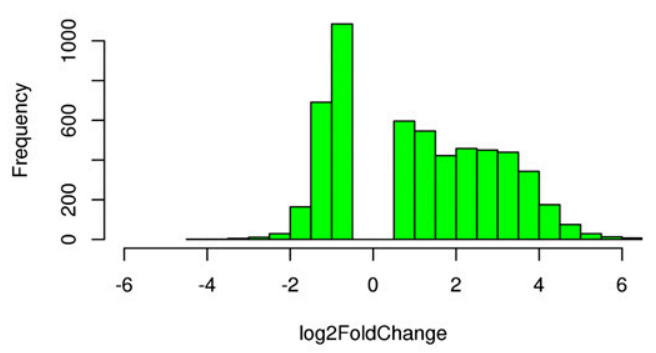

C

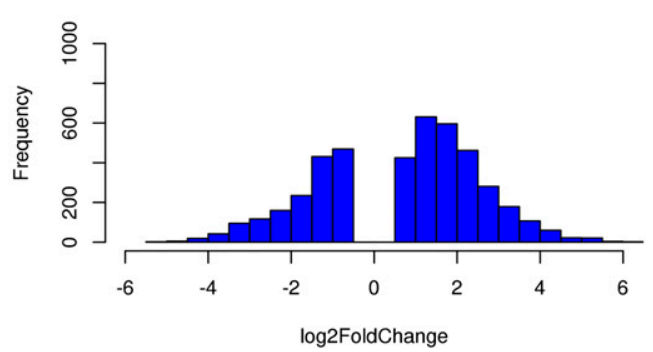

Figure 2. Expression differences between beta, islet, and nonbeta samples. $(A)$ Dot chart of top 10 highest expressed genes and their contribution to the nuclear transcriptome by cell type. (B) Histogram of $\log _{2}$ Fold Change (islet/beta) for differentially expressed genes (10\% FDR) in islets and beta cells. (C) Histogram of $\log _{2}$ Fold Change (nonbeta/beta) for differentially expressed genes (10\% FDR) in nonbeta and beta cells.

teins) central to both intercellular and cell-environment communication (Danzer et al. 2012), secretory proteins (signal peptide), and extracellular matrix components.

The two gene sets ("beta" and "nonbeta") offer a more accurate depiction of the transcripts that define the molecular identity of the major islet cell types: Table 3 shows the top 30 beta and nonbeta cell enriched transcripts, respectively, in descending order

Table 1. Differentially expressed (DE) genes and exon links between beta cells, islets, and nonbetas

(A) Differentially expressed genes (10\% FDR)—19,975 genes tested

\begin{tabular}{lccc}
\hline & $\begin{array}{c}\text { Beta } \\
\text { overexpressed }\end{array}$ & $\begin{array}{c}\text { Beta } \\
\text { underexpressed }\end{array}$ & Total \\
\hline Islet & 1987 & 3568 & 5555 \\
Nonbeta & 1583 & 2797 & 4380
\end{tabular}

(B) Differentially expressed exon links (10\% FDR), corresponding gene counts, and subset of candidate alternatively spliced genes (genes not DE but having DE links) -12,334 genes tested

\begin{tabular}{lccr}
\hline & $\begin{array}{c}\text { Beta } \\
\text { overexpressed }\end{array}$ & $\begin{array}{c}\text { Beta } \\
\text { underexpressed }\end{array}$ & Total \\
\hline Islet & & & \\
$\quad$ Links & 11,843 & 16,340 & 28,183 \\
$\quad$ Gene count & 2130 & 2964 & 5072 \\
$\quad \begin{array}{l}\text { Gene count excluding } \\
\text { DE genes }\end{array}$ & & 2167 \\
$\begin{array}{l}\text { Nonbeta } \\
\text { Links }\end{array}$ & 5688 & 10,238 & 15,926 \\
Gene count & 1199 & 1844 & 3025 \\
Gene count excluding DE genes & & 998 \\
\hline
\end{tabular}

Pairwise DESeq results (beta versus islet, beta versus nonbeta) of significant changes in magnitude of expression of genes (A), exon links (B) (reads spanning exon junctions). of RPKM and fold change (RPKM > 1 cutoff was used). This approach successfully filters out highly expressed genes in contaminating cell types (e.g., SST, GCG from somatostatin, and glucagon cells contaminating the beta cell population), otherwise mistaken as key players in the expression signature of beta cells. In addition to known beta-cell-specific transcripts (INS, IGF2, PDX1) we highlight further targets, some featured already in a microarray analysis of sorted islet cells (Dorrell et al. 2011b), e.g., RGS16, negative regulator of G-protein signaling, involved in endocrine pancreas development and re-expressed in adult cells in response to GLP-1 (Villasenor et al. 2010); ADCYAP1, pituitary adenylate cyclase activating polypeptide 1 , involved in insulin secretion and beta cell regeneration/proliferation (Sakurai et al. 2011); $H A D H$, hydroxyacyl-CoA dehydrogenase, negative regulator of insulin secretion (Hardy et al. 2007) associated with Alzheimer's (Nicolls et al. 2003), which is in turn associated with diabetes. Many other genes however have not been described before in the context of beta cells, including: NPTX2, neuronal pentraxin 2, found in neuronal cells and gliomas but also shown to be frequently downregulated in pancreatic cancers (Zhang et al. 2012); TSPAN1, tetraspanin 1 , which can associate with alpha6.beta1 integrin and promote $F A K$ phosphorylation (Huang et al. 2008) shown by us to be involved in insulin secretion (Rondas et al. 2011); GPM6A, neuronal membrane glycoprotein of unknown function but identified as a beta cell marker in sorted mouse islet cells (Dorrell et al. 2011a); BMP5, bone morphogenic protein 5, implicated in pancreas and fetal beta cell development (Jiang et al. 2002); and P2RY1, purinergic receptor through which ADP and ATP modulate insulin secretion (Fernandez-Alvarez et al. 2001).

Alternative splicing (AS), a common feature of most ( $94 \%)$ eukaryotic genes contributing to tissue specificity (Pan et al. 2008) is also significantly enriched in beta-cell-specific genes $(N=200$ genes, 1.22-fold enrichment). To assess the extent of AS between

\section{Genome Research www.genome.org}


Table 2. Functional characteristics of beta-cell-specific and -nonspecific genes in islet context

\begin{tabular}{|c|c|c|c|c|c|c|c|}
\hline \multicolumn{4}{|c|}{ Beta-cell-specific genes in islet context $(N=526)$} & \multicolumn{4}{|c|}{ Beta-cell-nonspecific genes in islet context $(N=614)$} \\
\hline Term & $\begin{array}{l}\text { Benjamini } \\
P \text {-value }\end{array}$ & Count & $\begin{array}{c}\text { Fold } \\
\text { enrichment }\end{array}$ & Term & $\begin{array}{l}\text { Benjamini } \\
P \text {-value }\end{array}$ & Count & $\begin{array}{l}\text { Fold } \\
\text { enrichment }\end{array}$ \\
\hline Neuron projection & $1.13 \times 10^{-5}$ & 26 & 3.53 & Glycoprotein & $5.26 \times 10^{-14}$ & 207 & 1.70 \\
\hline Axon & $1.35 \times 10^{-5}$ & 18 & 5.26 & Signal & $5.26 \times 10^{-14}$ & 169 & 1.84 \\
\hline Cell projection & $5.33 \times 10^{-5}$ & 37 & 2.47 & Signal peptide & $7.64 \times 10^{-13}$ & 169 & 1.83 \\
\hline Synapse & $5.79 \times 10^{-5}$ & 25 & 3.27 & $\begin{array}{l}\text { Glycosylation site:N-linked } \\
(\text { GlcNAc...) }\end{array}$ & $2.58 \times 10^{-11}$ & 194 & 1.65 \\
\hline Synapse part & $7.50 \times 10^{-5}$ & 20 & 3.78 & Secreted & $5.77 \times 10^{-10}$ & 99 & 2.07 \\
\hline $\begin{array}{l}\text { Passive transmembrane transporter } \\
\text { activity }\end{array}$ & $1.77 \times 10^{-4}$ & 27 & 3.02 & Disulfide bond & $1.50 \times 10^{-8}$ & 140 & 1.69 \\
\hline Substrate specific channel activity & $2.26 \times 10^{-4}$ & 26 & 3.02 & Extracellular region part & $1.18 \times 10^{-6}$ & 67 & 2.14 \\
\hline Intrinsic to membrane & $2.29 \times 10^{-4}$ & 155 & 1.31 & Extracellular region & $2.42 \times 10^{-6}$ & 110 & 1.68 \\
\hline Ion channel activity & $2.59 \times 10^{-4}$ & 26 & 3.11 & Plasma & $4.64 \times 10^{-6}$ & 16 & 6.08 \\
\hline $\begin{array}{l}\text { Metal ion transmembrane transporter } \\
\text { activity }\end{array}$ & $2.68 \times 10^{-4}$ & 23 & 3.24 & Response to wounding & $7.07 \times 10^{-5}$ & 42 & 2.62 \\
\hline Integral to membrane & $3.22 \times 10^{-4}$ & 150 & 1.32 & Membrane & $8.91 \times 10^{-5}$ & 230 & 1.30 \\
\hline Channel activity & $5.08 \times 10^{-4}$ & 27 & 3.03 & Extracellular matrix & $9.77 \times 10^{-5}$ & 23 & 3.37 \\
\hline Ionic channel & $7.98 \times 10^{-4}$ & 23 & 3.30 & Extracellular space & $2.30 \times 10^{-4}$ & 47 & 2.11 \\
\hline Gated channel activity & $1.15 \times 10^{-3}$ & 21 & 3.13 & Plasma membrane part & $2.51 \times 10^{-4}$ & 109 & 1.52 \\
\hline Calcium ion binding & $1.18 \times 10^{-3}$ & 41 & 2.06 & Growth factor & $2.65 \times 10^{-4}$ & 16 & 4.32 \\
\hline Ligand-gated channel activity & $1.26 \times 10^{-3}$ & 13 & 4.73 & Transmembrane & $5.07 \times 10^{-4}$ & 186 & 1.32 \\
\hline Ligand-gated ion channel activity & $1.26 \times 10^{-3}$ & 13 & 4.73 & Polymorphism & $6.75 \times 10^{-4}$ & 374 & 1.14 \\
\hline Cation channel activity & $1.74 \times 10^{-3}$ & 19 & 3.19 & $\begin{array}{l}\text { Proteinaceous extracellular } \\
\text { matrix }\end{array}$ & $1.06 \times 10^{-3}$ & 27 & 2.59 \\
\hline lon transport & $2.19 \times 10^{-3}$ & 31 & 2.44 & Extracellular matrix & $1.19 \times 10^{-3}$ & 28 & 2.49 \\
\hline Glycoprotein & $2.35 \times 10^{-3}$ & 131 & 1.38 & Cell fraction & $1.22 \times 10^{-3}$ & 61 & 1.73 \\
\hline
\end{tabular}

Top 20 most significantly overrepresented functional annotation terms (DAVID).

islet cell types, we quantified all the exon links (reads spanning pairs of exons only) (H Ongen and ET Dermitzakis, unpubl.) in the beta, islet and nonbeta preparations and tested for differential expression, those exon pairs making a minimum of five links in at least $90 \%$ of the samples $(N=154,190)$. We find 28,183 DE links (10\% FDR) between beta and islets and 15,926 DE links between beta and nonbeta (Table 1B), corresponding to 5072 and 3025 genes, respectively. In 2167 and 998 of these genes, respectively, the DE links observed between the different islet cell types were not due to DE of the underlying genes. This implicates $8 \%-18 \%$ of the 12,334 genes thus tested as candidates for islet AS.

Mapping RNA-seq data to the genome fails to identify reads that span exon junctions. To quantify this effect in our data and discover potential unannotated transcripts, we constructed all possible 96-mer exon junctions for each gene (read length- $1=48$ bases on either side of the junction) and mapped all reads against them. On average, 2.9 million reads per beta cell sample did not map to the genome but were recovered by junction mapping (Supplemental Table 3), a small percentage $(\sim 1.36 \%)$ of the total number of reads. Nevertheless, we used these together with reads mapping better to exon junctions than the genome (better mapping quality and larger alignment length) to assess the percentage of previously unobserved junctions. We identified 46,096 junctions in 7717 genes covered by at least 10 good quality reads (map quality greater than 10) in all beta cell samples, 657 of which $(1.4 \%$ of all junctions) were not present in the transcriptome annotation (GENCODE v10). These correspond to 465 genes enriched for posttranslational functions (acetylation: $8.9 \times 10^{-27}$; phosphoprotein: $1.3 \times 10^{-17}$; ribosome: $1.6 \times 10^{-14}$ ) and possibly containing new beta-cell-specific transcripts.

We next aimed to study the genetic control of the variation in beta cell and islet gene expression by integrating genome sequence level information when available. We extracted DNA from seven of the donors and sequenced them to medium $(16 \times)$ coverage (Supplemental Fig. 1b). The reads were aligned to the hg19 reference genome with BWA, we applied GATK (McKenna et al. 2010) base quality score recalibration, indel realignment and duplicate removal, followed by single nucleotide polymorphism (SNP) discovery and genotyping across all seven samples, simultaneously using variant quality score recalibration (DePristo et al. 2011). Subsequently, we imputed the variants on the 1000 Genomes reference panel and phased them with BEAGLE 3.3.2 (Browning and Browning 2007), resulting in 5,748,462 good confidence autosomal SNPs for analysis. For each individual, we tested the subset of heterozygous SNPs with good coverage in the RNA-seq data (beta, islet, and nonbeta) for allele-specific expression (ASE) (median 23,358 sites per sample). On average, we observe that $9.3 \%$ of the heterozygous sites tested show significant ASE at 10\% FDR (median 2626 sites per sample) (Supplemental Fig. 4). These correspond to a median of 1742 genes of 6756 genes tested per individual, harboring at least one ASE SNP (equivalent to $59.18 \%$ of the total number of tested genes in all samples). A subset of these genes are of particular functional interest, having been linked to diabetes in GWAS: 15 of 23 T1D genes tested show ASE, 20 of 28 T2D genes and 15 of 18 genes associated with fasting glucose or insulin levels. Except for the subset of genes associated to fasting glucose or insulin (Fisher's $P$-value 0.02), this enrichment was not statistically significant. A substantial number of diabetes susceptibility genes are however expressed in beta cells, corroborating the GWAS predictions (32/40 T1D, 37/39 T2D, 19/20 glucose or insulin levels) (Supplemental Fig. 5). Several of these $(N=9)$ are in fact betacell-specific transcripts: T1D-associated INS, imprinted MEG3-DLK1, GLIS3; T2D-associated VEGFA, SLC3OA8; fasting glucose/insulin levels-associated $A D R A 2 A, G 6 P C 2, S L C 2 A 2$. Interestingly, two other genes, SH2B3 (T1D-linked) and IRS1 (T2D-linked) are significantly 
Table 3. Top 30 genes enriched in the beta cell population and nonbeta cell population ordered by RPKM and fold change, respectively

\begin{tabular}{|c|c|c|c|c|c|c|c|}
\hline \multicolumn{4}{|c|}{ Top 30 beta cell enriched genes } & \multicolumn{4}{|c|}{ Top 30 nonbeta cell enriched genes } \\
\hline \multicolumn{2}{|c|}{ Ordered by RPKM } & \multicolumn{2}{|c|}{ Ordered by fold change } & \multicolumn{2}{|c|}{ Ordered by RPKM } & \multicolumn{2}{|c|}{ Ordered by fold change } \\
\hline Gene & Beta RPKM & Gene & $\begin{array}{l}\text { Fold change } \\
\text { Beta/nonbeta }\end{array}$ & Gene & Nonbeta RPKM & Gene & $\begin{array}{l}\text { Fold change } \\
\text { Nonbeta/beta }\end{array}$ \\
\hline INS & $131,369.34$ & INS-IGF2 & 20.17 & GCG & $37,234.91$ & IRX2 & 23.79 \\
\hline INS-IGF2 & $36,383.24$ & IGF2 & 19.99 & TTR & $12,418.51$ & GCG & 22.33 \\
\hline IGF2 & 7121.83 & INS & 18.88 & CLU & 895.89 & LOXL4 & 18.31 \\
\hline SLC30A8 & 393.03 & CAPN13 & 16.71 & GC & 415.26 & GC & 16.89 \\
\hline G6PC2 & 292.68 & WSCD2 & 14.84 & SERPINA1 & 410.05 & FAP & 16.55 \\
\hline DLK1 & 276.55 & NPTX2 & 13.98 & MUC13 & 220.59 & PAPPA2 & 15.66 \\
\hline ERO1LB & 272.02 & RGS16 & 13.07 & PTP4A3 & 176.18 & ARX & 13.89 \\
\hline PCSK1 & 207.63 & ADCYAP1 & 12.18 & TM4SF4 & 171.99 & MUC13 & 13.57 \\
\hline NPTX2 & 200.05 & $\mathrm{HADH}$ & 11.50 & TMEM176A & 144.97 & F10 & 13.07 \\
\hline SCD & 166.61 & TSPAN1 & 11.19 & EZR & 124.04 & VSTM2L & 12.88 \\
\hline VEGFA & 164.97 & ASB9 & 11.12 & TPM4 & 116.04 & FEV & 12.75 \\
\hline $\mathrm{HADH}$ & 163.95 & DLK1 & 10.88 & CRYBA2 & 113.63 & IGFBP2 & 12.20 \\
\hline EIF4A2 & 157.07 & GPM6A & 10.84 & IGFBP2 & 110.25 & MYO10 & 11.94 \\
\hline TIMP2 & 145.81 & GLP1R & 10.48 & COTL1 & 95.74 & KCNJ3 & 11.50 \\
\hline MEG3 & 140.99 & BMP5 & 10.15 & LOXL4 & 94.37 & $\mathrm{APOH}$ & 11.44 \\
\hline UCHL1 & 136.25 & P2RY1 & 9.99 & TMEM176B & 81.95 & TM4SF4 & 9.80 \\
\hline SYT13 & 122.22 & ENTPD3 & 9.96 & F10 & 79.35 & KCTD12 & 9.78 \\
\hline PDX1 & 119.25 & MEG3 & 9.28 & KCTD12 & 77.78 & $T T R$ & 9.62 \\
\hline ADCYAP1 & 99.62 & CYYR1 & 8.65 & $\mathrm{APOH}$ & 73.74 & SEPT9 & 9.21 \\
\hline GAD2 & 96.59 & VAT1L & 8.53 & LSR & 72.98 & NPNT & 9.01 \\
\hline ALCAM & 95.79 & C8orf47 & 8.38 & C19orf77 & 67.72 & FOSL2 & 8.61 \\
\hline NKX6-1 & 91.78 & PDX1 & 8.27 & FOSL2 & 64.69 & MAMLD1 & 8.31 \\
\hline GJD2 & 87.30 & SLC17A6 & 8.12 & LMNA & 58.09 & SERPINA1 & 7.93 \\
\hline TSC22D1 & 86.05 & O3FAR1 & 8.07 & IRX2 & 56.92 & SMOC1 & 7.69 \\
\hline CASR & 79.54 & SCD5 & 8.05 & PAPPA2 & 51.76 & FXYD5 & 7.59 \\
\hline SLC6A6 & 77.41 & OLFM1 & 8.05 & ARX & 50.97 & NEDD9 & 7.49 \\
\hline RP11-713B9.1 & 70.46 & SLC35D3 & 7.95 & VWA1 & 50.20 & KBTBD10 & 6.96 \\
\hline BTG3 & 64.76 & FAM115C & 7.90 & SMOC1 & 40.44 & AHNAK & 6.60 \\
\hline CABP7 & 59.66 & TMEM150C & 7.89 & RAP1GAP & 40.29 & LBH & 6.36 \\
\hline SORL1 & 56.36 & CASR & 7.78 & KBTBD10 & 39.63 & DUSP4 & 6.20 \\
\hline
\end{tabular}

Table contains genes with RPKM > 1 consistently and significantly overexpressed in beta cells (order of expression: beta $>$ islet $>$ nonbeta) and nonbeta cells, respectively (order of expression: nonbeta $>$ islet $>$ beta), ordered in descending order of RPKM and fold change.

overexpressed in the nonbeta population-given our data, it is tempting to speculate that alpha cell misregulation would be detrimental in these cases. In some instances, we find evidence of significant allelic imbalance for the exact GWAS SNPs reported, e.g., rs5215 (KCNJ11), a missense SNP associated to T2D risk is also an ASE variant in four beta cell samples, rs689 (INS), associated to T1D autoantibodies is an ASE SNP in four samples, and rs11558471 (SLC3OA8) associated with fasting glucose-related traits and proinsulin levels is an ASE SNP in 1 sample (the remaining samples were homozygous for the respective SNPs, hence could not be tested for ASE). These observations suggest that a subset of diabetes-associated loci could contribute to disease progression via a change in beta cell gene expression.

As expected for true positive ASE sites, the direction of effect is almost always consistent for the same individual between different tissues (Fig. 3). The high overall concordance between beta cell and islet expression data noted before is recapitulated at the sequence level. Taking difference of power into account (using pi1 to measure sharing of statistical significance), we estimate a median $89.11 \%$ enrichment of significant beta cell ASE $P$-values in the islet and a $88.11 \%$ median enrichment vice versa (significant islet ASE in the beta cell). One of the explanations for the ASE events discovered could be the presence of nearby regulatory variants (expression quantitative trait loci [eQTLs]). The current sample size is prohibitively small for eQTL discovery; however, in an effort to find those potentially active in beta cells, we integrated ASE sites with eQTLs discovered in the best-powered multitissue data set to date (MuTHER) (Grundberg et al. 2012), which reported 3148 eQTLs in fat, 3953 eQTLs in lymphoblastoid cell lines (LCLs), and 2515 eQTLs in skin. For each gene and tissue, we phased the top eQTL SNP and top ASE SNP per individual, being thus able to test in our data 1806 of the eQTLs discovered in fat, 2272 eQTLs discovered in LCLs, and 1400 eQTLs discovered in skin. We observe a significantly greater deviation from the expected reference/total allelic ratio (0.5) in individuals heterozygous for the eQTLs compared to homozygotes (Mann-Whitney $P$-value $<6.18 \times 10^{-11}$ ), indicating that a proportion of these regulatory variants are also active in beta cells (Fig. 4). To shortlist them, we selected cases in which the direction of the eQTL effect was in agreement with the significant (ASE $P$-value $<0.01$ ) allelic imbalance prediction. We thus report 254 candidate beta eQTL genes using the data from fat cells, 294 genes from LCLs, and 198 from skin. Reassuringly, 303 of the 510 total genes detected as such $(59.4 \%)$ are shared in at least two of the MuTHER tissues, a significant enrichment (Fisher's $P$-value: $6.2 \times 10^{-10}$ ) over the $44.8 \%$ eQTL sharing we began with. This suggests that these eQTLs are not highly tissue specific and therefore likely to be also present in beta cells.

\section{Discussion}

Pancreatic islets are essential for regulating and maintaining glucose homeostasis. This functional role is fulfilled by at least five 
pi $1=0.8835$

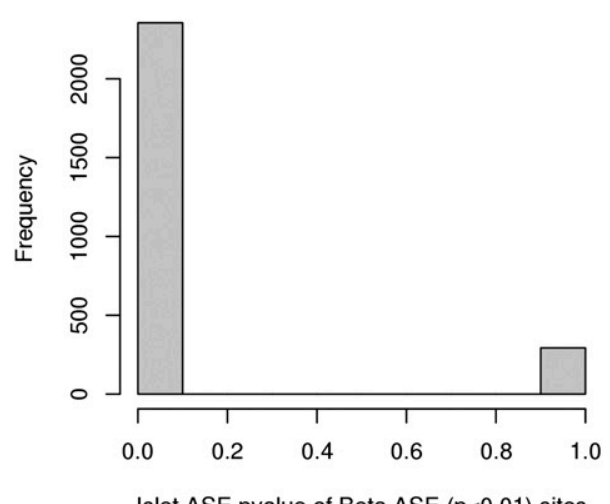

Islet ASE pvalue of Beta ASE $(p<0.01)$ sites

pi $1=0.8958$

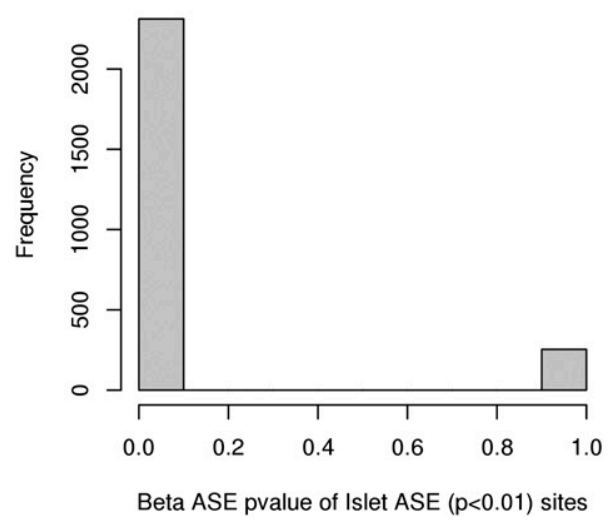

ASE $p<0.01$ sites

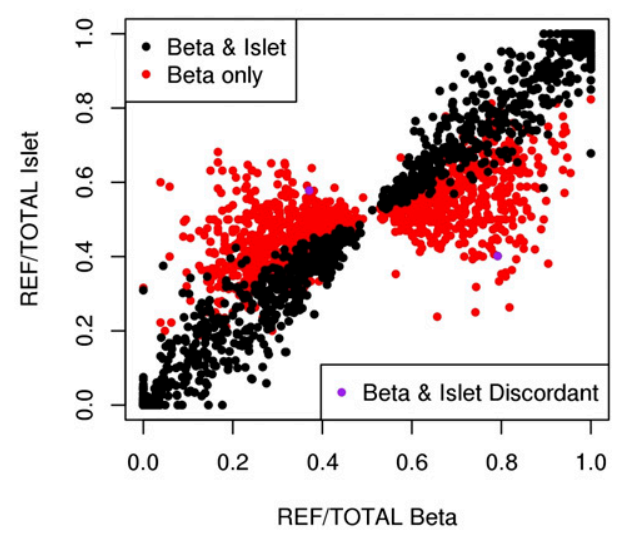

ASE $p<0.01$ sites

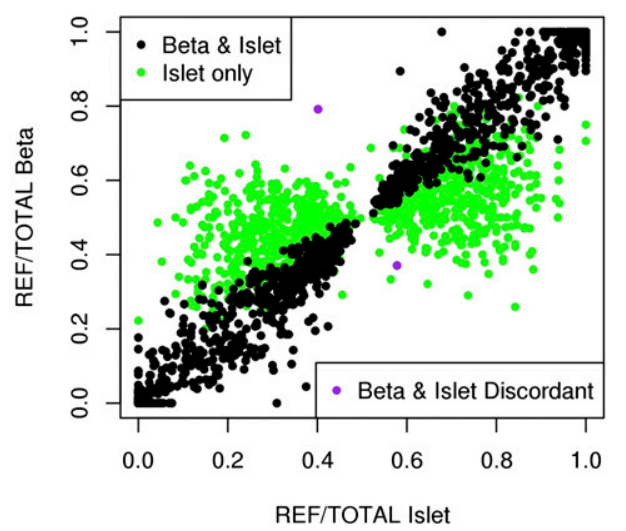

Figure 3. Sharing of significant ASE effects between beta cells and islets (sample P775). Left panel histograms show the enrichment (pi1) of significant ASE $P$-values in the islets for ASE sites discovered in beta cells and vice versa (beta cell ASE $P$-values of ASE sites discovered in islets). Right panel scatterplots display the direction of ASE effects (ratio of reference allele count to the total number of reads covering that site) between the two cell types, almost always in concordance.

distinct hormone-producing cell types, which act differently but synergistically to help maintain appropriate glycemic levels in healthy individuals. Therefore, uncovering the molecular identity of ideally each of these cell types would ensure a better understanding of the mechanisms through which they become defective in diabetic patients. We present here the first ever unbiased transcriptome analysis of the differentiated human beta cell, the most abundant pancreatic islet cell type that secretes insulin and is severely affected in common forms of diabetes. The advanced cell sorting protocol, immediate RNA extraction, and unprecedented sequencing resolution makes this unique data set the most faithful representation of the human beta cell transcriptome to date. Importantly, we compare this to the expression profile of whole islets and beta cell depleted islet preparations ("nonbeta"), uncovering unique beta-cell-specific features in contrast to other pancreatic endocrine cell types. In the absence of the three-way beta-isletnonbeta transcriptome comparison enabled by our study design, such discoveries would remain concealed.

Overall, we observe that islets are a good proxy for beta cell gene expression, provided they are of high enough purity (>85\%). However, important biological insights into cell-type-specific expression signatures are revealed when comparing detailed RNA profiles of purified beta cells with those of whole islets or "nonbeta" cells. We find substantial evidence for differential expression between beta cells and islets, with more than 5000 genes showing significant changes in transcript abundance between the two. Genes consistently overexpressed in beta cells are enriched for neuron-like properties, a similarity suspected earlier that we now independently confirm. These important biological insights would not be revealed by islet expression studies alone. Further, we corroborate current GWAS results by reporting beta cell expression of a large number of genes (80\%-95\%) previously associated with type 1 or type 2 diabetes. Differential splicing is also present among islet cell types, with up to $18 \%$ of genes undergoing this process in our data. Altogether, this draws attention to the general limitations of analyzing a mixture of cell populations as opposed to preparations highly enriched for a single cell type of interest.

Finally, we describe here the first set of genetic variants controlling interindividual variability in beta cell gene expression. Despite the small sample size and consequent limited number of informative genetic variants, we are able to report allelic and genotypic expression differences, offering a first glance at the genetics behind human beta cell function. Our evidence for diabetes-associated loci expressed in an allele-specific manner 
fat

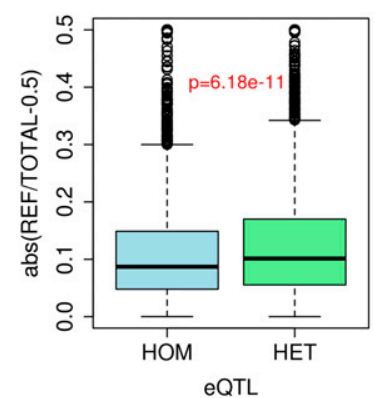

fat

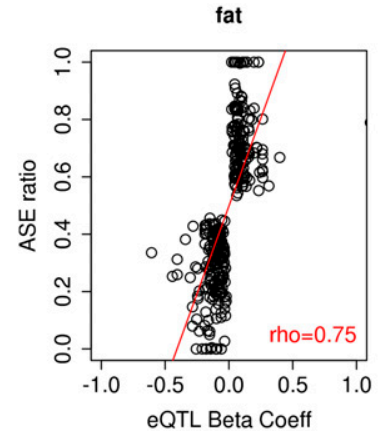

$|c|$

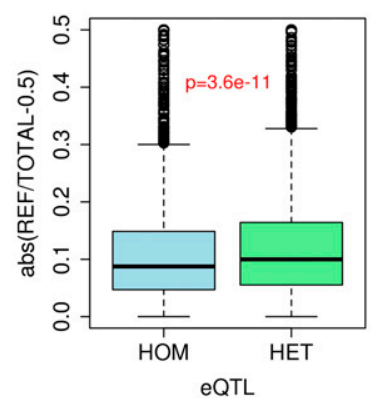

IcI

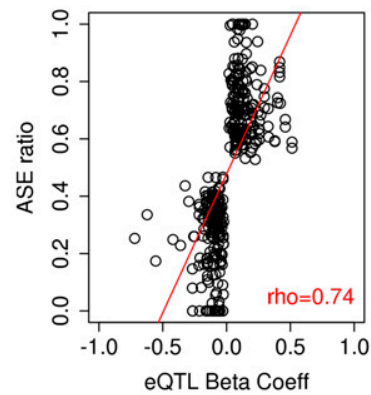

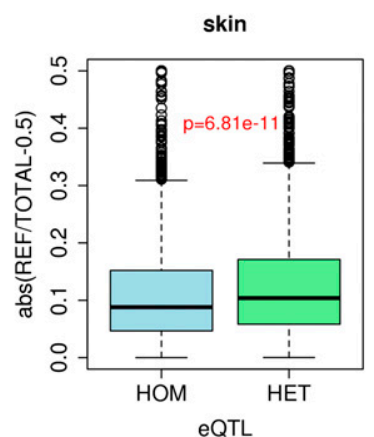

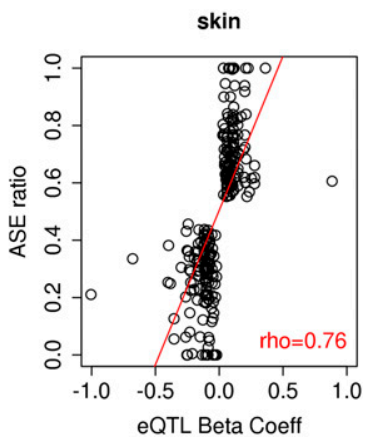

Figure 4. Candidate beta cell cis eQTLs discovered initially in other tissues (fat, LCL, skin). Top panel boxplots show the deviations of allelic ratios (reference/total) from the expected 0.5 in ASE individuals grouped by eQTL genotype, with heterozygotes having markedly higher effects on ASE ratios compared to homozygotes. Bottom scatterplots show the beta coefficients of the MuTHER eQTLs and the corresponding beta ASE ratios for the selected candidate beta cell regulatory variants.

flags potential contributions to disease effects by genetically or epigenetically driven expression changes in beta cells. These are encouraging results, which remain to be refined and augmented by a much-awaited beta cell eQTL study.

Taken together, the data will serve the diabetes research community manifold ways, including toward the validation of future stem-cell derived or other surrogate beta cells for research purposes or regenerative medicine.

\section{Methods}

\section{Sample collection}

Islets isolated from cadaveric pancreas were provided by the Cell Isolation and Transplant Center, Department of Surgery, Geneva University Hospital (Drs. T. Berney and D. Bosco) through the Juvenile Diabetes Research Foundation (JDRF) award 31-2008-416 (ECIT Islet for Basic Research Program).

\section{Beta cell sorting}

Islets were dispersed into single cells, stained with Newport Green, and sorted into "beta" and "nonbeta" populations as described previously (Parnaud et al. 2008). The proportion of beta (insulin), alpha (glucagon), and delta (somatostatin) cells in each population (as percentage of total cells) was determined by immunofluorescence.

\section{RNA extraction}

Sorted beta cells, nonbeta cells, and islets were centrifuged; the supernatant was removed; and the pellet disrupted in RLT buffer (RNeasy, Qiagen). Total RNA was prepared according to the standard RNeasy protocol.

\section{DNA extraction}

DNA was prepared according to the standard DNeasy (Qiagen) protocol.

\section{Library preparation and sequencing}

Total RNA and genomic DNA libraries were constructed following customary Illumina TruSeq protocols for nextgeneration sequencing. PolyA-selected mRNAs were purified, size-fractioned, and subsequently converted to singlestranded cDNA by random hexamer priming. Following second-strand synthesis, double-stranded cDNAs were blunt-end fragmented and indexed using adapter ligation, after which they were amplified and sequenced according to protocol. RNA libraries were 49-bp paired-end sequenced with one or a maximum of two samples per HiSeq 2000 lane. DNA libraries for whole-genome sequencing were constructed similarly, but starting directly from fragmented genomic DNA. The seven DNA libraries were pooled and sequenced in a total of nine HiSeq lanes each (one control lane of 49-bp pairedend reads and eight lanes of 95-bp pairedend sequencing). Standard quality checks for material degradation (Bioanalyzer, Agilent Technologies) and concentration (Qubit, Life Technologies) were done before and after library construction, ensuring that samples are suitable for sequencing.

\section{Read mapping}

Paired-end reads were mapped to the human genome (assembly version GRCh37/hg19) using BWA and allowing a maximum insert size of $500 \mathrm{~kb}$ (for instances when not enough good alignments could be used to infer the insert size distribution). RNA-seq reads were subsequently filtered for correct orientation of the mapped mate pairs with an insert size $<500 \mathrm{~kb}$ and a minimum mapping quality score of 10 .

\section{Expression quantification}

GENCODE annotation v10 was used to assign filtered reads to their corresponding exons and genes. We filtered the annotation to include transcribed biotypes (coding and noncoding), i.e., all protein coding genes, lincRNAs, processed transcripts, noncoding, polymorphic pseudogenes, transcribed processed pseudogenes, and transcribed unprocessed pseudogenes. For each gene, we processed the exons from all transcripts, which we quantified by taking into account only filtered reads as above, in which both mates of a pair map to exons of the same gene. The gene counts were incremented nonredundantly, i.e., reads overlapping two exons are counted once to the total count sum per gene. Resulting raw gene counts were normalized to gene length (sum of exons) and sequencing depth, i.e., reads per kilobase per million (RPKM) mapped reads.

\section{Differential expression analysis}

Raw gene/exon link counts were modeled using a negative binomial distribution and tested for differential gene expression

\section{Genome Research www.genome.org}


with the DESeq R package. Following author's guidelines, we estimated the size factor for each sample from the count data, followed by dispersion estimations for each gene/link. Subsequently, we tested for differential expression between conditions. We grouped all beta cell, islet, nonbeta, and the 18 different tissues (adipose, bladder, brain, cervix, colon, esophagus, heart, kidney, liver, lung, ovary, placenta, prostate, spleen, testes, thymus, thyroid, trachea) in separate conditions (beta, islet, nonbeta, other) and tested for differential expression: beta versus other, beta versus islet, and beta versus nonbeta. For the pairwise DESeq tests of islet-derived data only, we included islet purity as a covariate: We fitted two generalized linear models and compared them to see whether the purity factor improved the fit (i.e., had a significant effect). The $P$-values of the comparisons were then adjusted for multiple testing using the Benjamini-Hochberg method. Adjusted $P$-values $<0.1$ were considered significant. Introducing the islet purity as a covariate improved the fit in all cases.

\section{Quantification of exon links}

We made use of the paired-end nature of our RNA-seq experiment to relatively quantify splicing events. Specifically, we used filtered mate pairs (correctly paired and 10 minimum mapping quality) where one mate maps to one exon and the other mate to a different exon to count "links" between two exons. The first exon in a link was referred to as the "primary exon." Overlapping exons were grouped into "exon groups" and unique portions of each exon in an exon group were identified. These unique portions were subsequently used to assign reads to an exon.

\section{Generation of exon junctions}

We have constructed all possible 96-mer junctions (flanking sequence on either side $=$ read length -1 ) per gene using the transcriptome information in Ensembl v65 (Flicek et al. 2013). For each gene, we first kept track of all the unique start and end positions of all its exons. Subsequently, for nonredundant exon-exon pairs with valid positions (i.e., starting position of second exon greater than ending position of first exon in junction), we constructed every possible pairwise combination within the length limits of the exons involved. For junctions failing this criterion due to short exons on one or both ends, the sequence was expanded into flanking exons. For every valid junction, we recorded whether it forms part of any known transcript or not.

\section{Functional annotation analysis}

Gene functional analyses were performed using The Database for Annotation, Visualization and Integrated Discovery (DAVID) v6.7. We have used the Functional Annotation Chart tool to analyze our genes of interest (e.g., beta cell specific) in the context of the whole human genome. DAVID's default annotation categories were used and enrichment results sorted by significance of Benjamini corrected $P$-values.

\section{SNP calling}

Variant calling was performed with the Genome Analysis Toolkit (GATK) 1.5.31 following the Best Practice Variant Detection v3. Reads were aligned to the hg19 reference genome with BWA, and bam files from each lane were merged into one bam per sample. For each sample we removed duplicates, realigned the reads around known indels from the 1000 Genomes, applied GATK base quality score recalibration, followed then by SNP discovery and genotyping across all seven samples simultaneously with variant quality score recalibration. We used a confidence score threshold of 30 for variant detection and a minimum base quality of 17 to consider a base for calling. Good confidence (1\% FDR) SNP calls were then imputed on the 1000 Genomes reference panel and phased with BEAGLE 3.3.2.

\section{ASE analysis}

Allele-specific expression (ASE) analysis was performed perindividual on the subset of heterozygous sites with a minimum coverage of 16 reads per site filtered for mapping quality of 10 . Problematic positions were filtered out as follows to exclude sites susceptible to allelic mapping bias: (1) sites with UCSC 50-bp mapability less than one, implying that the 50-bp flanking region of the site is nonunique in the genome and collapsed repeat regions were excluded; and (2) simulated RNA-seq reads overlapping the site and showing $>5 \%$ difference in the mapping of reads that carry the reference or nonreference allele were also excluded. Next, we calculated the expected reference allele ratio for each individual by summing up reads across all sites separately for each SNP allele combination after down-sampling reads of sites in the top 25th coverage percentile in order to avoid the highest covered sites having a disproportionally large effect on the ratios. These expected REF/TOTAL ratios (in the range of 0.489-0.518) correct for the remaining slight genome-wide mapping bias in each individual. In a binomial test of the REF/NONREF allele counts, we used these individual-specific expected ratios to weight the occurrence of each allele and determine sites with $\geq 16$ reads in each individual deviating from the biased-corrected expected allelic ratios. We called sites in significant ASE if resulting $P$-values $<0.01$.

\section{eQTL integration}

We obtained cis eQTLs (1\% FDR) detected in the MuTHER study, where 856 female twins were profiled for gene expression with microarrays in three tissues: fat, lymphoblastoid cell lines (LCLs), and skin. These amounted to 3148 genes in fat, 3953 genes in LCLs, and 2515 genes in skin having cis eQTLs. We phased the top SNPs per gene in each of these data sets with top beta cell ASE sites for the same genes. We contrasted the beta cell allelic ratios for individuals heterozygous for the MuTHER eQTLs compared to homozygotes, expecting smaller effects in homozygotes, where the eQTL itself (if active also in beta cells) would have no effect on expression.

\section{Data access}

Genotype and sequence data have been deposited at the European Genome-phenome Archive (EGA; http://www.ebi.ac.uk/ega/), which is hosted by the European Bioinformatics Institute (EBI), under accession number EGAS00001000442. Anonymized sequence files (matching the reference genome) are freely and publicly available on our ftp server (ftp://jungle.unige.ch/) as well as Ensembl.

\section{Acknowledgments}

This work was supported by grant number 17-2011-284 from JDRF, the Innovative Medicines Initiative Joint Undertaking under grant agreement no. 115317 (DIRECT), and the Louis-Jeantet Foundation. We thank Stephane Dupuis, Ismael Padioleau, Alexandra 
Planchon, Luciana Romano, and Alisa Yurovsky for expert technical assistance.

\section{References}

Ahren B. 2000. Autonomic regulation of islet hormone secretionimplications for health and disease. Diabetologia 43: 393-410.

Anders S, Huber W. 2010. Differential expression analysis for sequence count data. Genome Biol 11: R106.

Barrett JC, Clayton DG, Concannon P, Akolkar B, Cooper JD, Erlich HA, Julier C, Morahan G, Nerup J, Nierras C, et al. 2009. Genome-wide association study and meta-analysis find that over 40 loci affect risk of type 1 diabetes. Nat Genet 41: 703-707.

Browning SR, Browning BL. 2007. Rapid and accurate haplotype phasing and missing-data inference for whole-genome association studies by use of localized haplotype clustering. Am J Hum Genet 81: 10841097.

Cabili MN, Trapnell C, Goff L, Koziol M, Tazon-Vega B, Regev A, Rinn JL. 2011. Integrative annotation of human large intergenic noncoding RNAs reveals global properties and specific subclasses. Genes Dev 25: 1915-1927.

Danzer C, Eckhardt K, Schmidt A, Fankhauser N, Ribrioux S, Wollscheid B, Müller L, Schiess R, Züllig R, Lehmann R, et al. 2012. Comprehensive description of the $\mathrm{N}$-glycoproteome of mouse pancreatic $\beta$-cells and human islets. J Proteome Res 11: 1598-1608.

DePristo MA, Banks E, Poplin R, Garimella KV, Maguire JR, Hartl C, Philippakis AA, del Angel G, Rivas MA, Hanna M, et al. 2011. framework for variation discovery and genotyping using nextgeneration DNA sequencing data. Nat Genet 43: 491-498.

Dimas AS, Deutsch S, Stranger BE, Montgomery SB, Borel C, Attar-Cohen H, Ingle C, Beazley C, Gutierrez Arcelus M, Sekowska M, et al. 2009. Common regulatory variation impacts gene expression in a cell typedependent manner. Science 325: 1246-1250.

Dorrell C, Grompe MT, Pan FC, Zhong Y, Canaday PS, Shultz LD, Greiner DL, Wright CV, Streeter PR, Grompe M. 2011a. Isolation of mouse pancreatic $\alpha, \beta$, duct and acinar populations with cell surface markers. Mol Cell Endocrinol 339: 144-150.

Dorrell C, Schug J, Lin CF, Canaday PS, Fox AJ, Smirnova O, Bonnah R, Streeter PR, Stoeckert CJ Jr, Kaestner KH, et al. 2011b. Transcriptomes of the major human pancreatic cell types. Diabetologia 54: 28322844.

Emilsson V, Thorleifsson G, Zhang B, Leonardson AS, Zink F, Zhu J, Carlson S, Helgason A, Walters GB, Gunnarsdottir S, et al. 2008. Genetics of gene expression and its effect on disease. Nature 452: 423-428.

Fernandez-Alvarez J, Hillaire-Buys D, Loubatières-Mariani MM, Gomis R, Petit P. 2001. P2 receptor agonists stimulate insulin release from human pancreatic islets. Pancreas 22: 69-71.

Flicek P, Ahmed I, Amode MR, Barrell D, Beal K, Brent S, Carvalho-Silva D, Clapham P, Coates G, Fairley S, et al. 2013. Ensembl 2013. Nucl Acids Res 41: D48-D55.

Gaulton KJ, Nammo T, Pasquali L, Simon JM, Giresi PG, Fogarty MP, Panhuis TM, Mieczkowski P, Secchi A, Bosco D, et al. 2010. A map of open chromatin in human pancreatic islets. Nat Genet 42: 255-259.

Grundberg E, Small KS, Hedman AK, Nica AC, Buil A, Keildson S, Bell JT, Yang TP, Meduri E, Barrett A, et al. 2012. Mapping cis- and transregulatory effects across multiple tissues in twins. Nat Genet 44: 10841089.

Hardy OT, Hohmeier HE, Becker TC, Manduchi E, Doliba NM, Gupta RK, White P, Stoeckert CJ Jr, Matschinsky FM, Newgard CB, et al. 2007. Functional genomics of the $\beta$-cell: short-chain 3-hydroxyacylcoenzyme A dehydrogenase regulates insulin secretion independent of $\mathrm{K}^{+}$currents. Mol Endocrinol 21: 765-773.

Harrow J, Denoeud F, Frankish A, Reymond A, Chen CK, Chrast J, Lagarde J, Gilbert JG, Storey R, Swarbreck D et al. 2006. GENCODE: Producing a reference annotation for ENCODE. Genome Biol 7 (Suppl 1): S4

Huang Y, Sook-Kim M, Ratovitski E. 2008. Midkine promotes tetraspaninintegrin interaction and induces FAK-Stat $1 \alpha$ pathway contributing to migration/invasiveness of human head and neck squamous cell carcinoma cells. Biochem Biophys Res Commun 377: 474-478.

Huang DW, Sherman BT, Lempicki RA. 2009. Systematic and integrative analysis of large gene lists using DAVID bioinformatics resources. Nat Protoc 4: 44-57.

Jiang FX, Stanley EG, Gonez LJ, Harrison LC. 2002. Bone morphogenetic proteins promote development of fetal pancreas epithelial colonies containing insulin-positive cells. J Cell Sci 115: 753-760.

Li H, Durbin R. 2009. Fast and accurate short read alignment with BurrowsWheeler transform. Bioinformatics 25: 1754-1760.
McKenna A, Hanna M, Banks E, Sivachenko A, Cibulskis K, Kernytsky A, Garimella K, Altshuler D, Gabriel S, Daly M, et al. 2010. The Genome Analysis Toolkit: A MapReduce framework for analyzing nextgeneration DNA sequencing data. Genome Res 20: 1297-1303.

Montgomery SB, Sammeth M, Gutierrez-Arcelus M, Lach RP, Ingle C, Nisbett J, Guigo R, Dermitzakis ET. 2010. Transcriptome genetics using second generation sequencing in a Caucasian population. Nature 464: 773777.

Morán I, Akerman I, van de Bunt M, Xie R, Benazra M, Nammo T, Arnes L, Nakic N, Garcia-Hurtado J, Rodríguez-Seguí S, et al. 2012. Human $\beta$ cell transcriptome analysis uncovers lncRNAs that are tissue-specific, dynamically regulated, and abnormally expressed in type 2 diabetes. Cell Metab 16: 435-448.

Morris AP, Voight BF, Teslovich TM, Ferreira T, Segre AV, Steinthorsdottir V, Strawbridge RJ, Khan H, Grallert H, Mahajan A, et al. 2012. Large-scale association analysis provides insights into the genetic architecture and pathophysiology of type 2 diabetes. Nat Genet 44: 981-990.

Mortazavi A, Williams BA, McCue K, Schaeffer L, Wold B. 2008. Mapping and quantifying mammalian transcriptomes by RNA-Seq. Nat Methods 5: $621-628$.

Nica AC, Montgomery SB, Dimas AS, Stranger BE, Beazley C, Barroso I, Dermitzakis ET. 2010. Candidate causal regulatory effects by integration of expression QTLs with complex trait genetic associations. PLoS Genet 6: e1000895.

Nica AC, Parts L, Glass D, Nisbet J, Barrett A, Sekowska M, Travers M, Potter S, Grundberg E, Small K, et al. 2011. The architecture of gene regulatory variation across multiple human tissues: The MuTHER study. PLoS Genet 7: e1002003.

Nicolls MR, D'Antonio JM, Hutton JC, Gill RG, Czwornog JL, Duncan MW. 2003. Proteomics as a tool for discovery: Proteins implicated in Alzheimer's disease are highly expressed in normal pancreatic islets. I Proteome Res 2: 199-205.

Pan Q, Shai O, Lee LJ, Frey BJ, Blencowe BJ. 2008. Deep surveying of alternative splicing complexity in the human transcriptome by highthroughput sequencing. Nat Genet 40: 1413-1415.

Parnaud G, Bosco D, Berney T, Pattou F, Kerr-Conte J, Donath MY, Bruun C, Mandrup-Poulsen T, Billestrup N, Halban PA. 2008. Proliferation of sorted human and rat $\beta$ cells. Diabetologia 51: $91-100$.

Pickrell JK, Marioni JC, Pai AA, Degner JF, Engelhardt BE, Nkadori E, Veyrieras JB, Stephens M, Gilad Y, Pritchard JK. 2010. Understanding mechanisms underlying human gene expression variation with RNA sequencing. Nature 464: 768-772.

Pisania A, Weir GC, O'Neil JJ, Omer A, Tchipashvili V, Lei J, Colton CK, Bonner-Weir S. 2010. Quantitative analysis of cell composition and purity of human pancreatic islet preparations. Lab Invest 90: 16611675 .

Price AL, Helgason A, Thorleifsson G, McCarroll SA, Kong A, Stefansson K. 2011. Single-tissue and cross-tissue heritability of gene expression via identity-by-descent in related or unrelated individuals. PLoS Genet 7: e1001317.

Rondas D, Tomas A, Halban PA. 2011. Focal adhesion remodeling is crucial for glucose-stimulated insulin secretion and involves activation of focal adhesion kinase and paxillin. Diabetes 60: 1146-1157.

Sakurai Y, Shintani N, Hayata A, Hashimoto H, Baba A. 2011. Trophic effects of PACAP on pancreatic islets: A mini-review. J Mol Neurosci 43: 3-7.

Saltiel AR, Kahn CR. 2001. Insulin signalling and the regulation of glucose and lipid metabolism. Nature 414: 799-806.

Schwartz MW, Woods SC, Porte D Jr, Seeley RJ, Baskin DG. 2000. Central nervous system control of food intake. Nature 404: 661-671.

Storey JD, Tibshirani R. 2003. Statistical significance for genomewide studies. Proc Natl Acad Sci 100: 9440-9445.

Stranger BE, Nica AC, Forrest MS, Dimas A, Bird CP, Beazley C, Ingle CE, Dunning M, Flicek P, Koller D, et al. 2007. Population genomics of human gene expression. Nat Genet 39: 1217-1224.

Thomas MF, Ansel KM. 2010. Construction of small RNA cDNA libraries for deep sequencing. Methods Mol Biol 667: 93-111.

Tisch R, McDevitt H. 1996. Insulin-dependent diabetes mellitus. Cell 85: 291-297.

Villasenor A, Wang ZV, Rivera LB, Ocal O, Asterholm IW, Scherer PE, Brekken RA, Cleaver O, Wilkie TM. 2010. Rgs16 and Rgs8 in embryonic endocrine pancreas and mouse models of diabetes. Dis Model Mech 3: 567-580.

Zhang L, Gao J, Li Z, Gong Y. 2012. Neuronal pentraxin II (NPTX2) is frequently down-regulated by promoter hypermethylation in pancreatic cancers. Dig Dis Sci 57: 2608-2614.

Received October 12, 2012; accepted in revised form May 2, 2013.

\section{Genome Research}

www.genome.org 


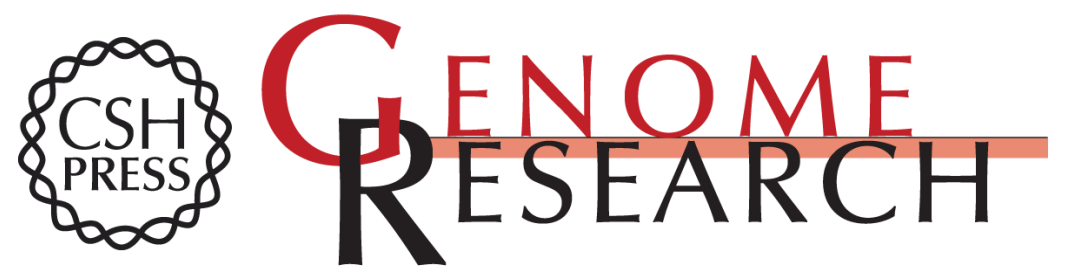

\section{Cell-type, allelic, and genetic signatures in the human pancreatic beta cell transcriptome}

Alexandra C. Nica, Halit Ongen, Jean-Claude Irminger, et al.

Genome Res. 2013 23: 1554-1562 originally published online May 28, 2013

Access the most recent version at doi:10.1101/gr.150706.112

Supplemental Material

References

Creative

Commons

License

Email Alerting Service
http://genome.cshlp.org/content/suppl/2013/06/25/gr.150706.112.DC1

This article cites 44 articles, 7 of which can be accessed free at: http://genome.cshlp.org/content/23/9/1554.full.html\#ref-list-1

This article is distributed exclusively by Cold Spring Harbor Laboratory Press for the first six months after the full-issue publication date (see

http://genome.cshlp.org/site/misc/terms.xhtml). After six months, it is available under a Creative Commons License (Attribution-NonCommercial 3.0 Unported), as described at http://creativecommons.org/licenses/by-nc/3.0/.

Receive free email alerts when new articles cite this article - sign up in the box at the top right corner of the article or click here.

\section{Affordable, Accurate Sequencing.}

To subscribe to Genome Research go to:

https://genome.cshlp.org/subscriptions 